Bull. Austral. Math. Soc.

$90 \mathrm{c} 29,90 \mathrm{c} 30$

VoL. 67 (2003) [429-443]

\title{
S-STRICTLY QUASI-CONCAVE VECTOR MAXIMISATION
}

\author{
Hong-Bin Dong, Xun-Hua Gong, Shou-Yang Wang and Luis Coladas
}

In this paper, we discuss the relationship among the concepts of an $S$-strictly quasiconcave vector-valued function introduced by Benson and Sun, a $C$-strongly quasiconcave vector-valued function and a $C$-strictly quasiconcave vector-valued function in a topological vector space with a lattice ordering. We generalise a main result obtained by Benson and Sun about the closedness of an efficient solution set in multiple objective programming. We prove that an efficient solution set is closed and connected when the objective function is a continuous $S$-strictly quasiconcave vectorvalued function, the objective space is a topological vector lattice and the ordering cone has a nonempty interior.

\section{INTRODUCTION}

In vector optimisation, the closedness and connectedness of an efficient solution set is an interesting topic (see [2]). But very few researchers have studied the closedness of an efficient solution set for a vector optimisation problem (see $[5,11,13]$ ).

On the other hand, many authors investigated the following open problem: whether the efficient solution set is connected when the objective function $f=\left(f_{1}, f_{2}, \ldots, f_{n}\right)$ is strictly quasiconcave (that is, for each $i \in\{1,2, \ldots, n\}$, the real-valued function $f_{i}$ is strictly quasiconcave) on a convex compact set $A$ (see $[1,3,4,8,12])$.

In an infinite dimensional space, Fu and Zhou $[6,7]$ investigated the connectedness of the efficient solution set for a $C$-strictly quasiconcave vector optimisation problem under the condition that the efficient solution set is closed. Fu and Zhou [7] gave an example to illustrate that even if the objective function is continuous and $C$-strictly quasiconcave and the feasible set is compact, the efficient solution set is not necessarily connected.

Recently, Benson and Sun [2] introduced a new concept for a strictly quasiconcave vector-valued function. This concept is an important tool for studying closedness and connectedness of the efficient solution set for a strictly quasiconcave vector optimisation problem.

Received 24th September, 2002

Supported by the Natural Science Foundation of Jiangxi Province, the National Natural Science Foundation of China, and the Lotus Program of Hunan Province.

Copyright Clearance Centre, Inc. Serial-fee code: 0004-9727/03 \$A2.00+0.00. 
In this paper, we discuss the relationship among the concepts of an $S$-strictly quasiconcave vector-valued function introduced by Benson and Sun [2], a $C$-strongly quasiconcave vector-valued function and a $C$-strictly quasiconcave vector-valued function in a topological vector space with a lattice ordering. We generalise a main result obtained by Benson and Sun about the closedness of an efficient solution set in multiple objective programming. We prove that an efficient solution set is closed and connected when the objective function is a continuous $S$-strictly quasiconcave vector-valued function, the objective space is a topological vector lattice and the ordering cone has a nonempty interior.

\section{Definitions AND Lemmas}

Let $X$ be a real topological vector space and $Y$ be a real ordered vector space whose partial order is introduced by a closed convex pointed cone $C$. We write

$$
y_{1} \leqslant y_{2} \quad \text { if and only if } \quad y_{2}-y_{1} \in C
$$

and

$$
y_{1}<y_{2} \text { if and only if } \quad y_{2}-y_{1} \in \operatorname{int} C \text {, }
$$

for any $y_{1}, y_{2} \in Y$.

For any $y_{1}, y_{2} \in Y$, let $\inf \left\{y_{1}, y_{2}\right\}$ denote the infimum of $y_{1}, y_{2}$, that is, $y_{i}$ $\in \inf \left\{y_{1}, y_{2}\right\}+C, i=1,2$, and if $y \in Y$ with $y_{i} \in y+C, i=1,2$, then $\inf \left\{y_{1}, y_{2}\right\} \in y+C$. In other words, $\inf \left\{y_{1}, y_{2}\right\}$ is the largest lower bound of the set $\left\{y_{1}, y_{2}\right\}$. Since $C$ is a pointed cone, $\inf \left\{y_{1}, y_{2}\right\}$ is unique. Let $\sup \left\{y_{1}, y_{2}\right\}$ denote the supremum of $y_{1}, y_{2}$. An ordered vector space $Y$ is called a vector lattice if $\inf \left\{y_{1}, y_{2}\right\}$ and $\sup \left\{y_{1}, y_{2}\right\}$ exist for each pair $\left(y_{1}, y_{2}\right) \in Y \times Y$.

Define $|y|=\sup \{y,-y\}$. A subset $B$ of a vector lattice $Y$ is said to be solid if $b \in B$ and $|y| \leqslant|b|$ imply that $y \in B$. Let $Y$ be a topological vector space with a lattice ordering. We say that $Y$ is locally solid if the solid neighbourhoods of 0 form a local base. A Hausdorff topological vector space $Y$ is said to be a topological vector lattice if $Y$ is locally solid (see $[9,10]$ ).

Throughout the paper, we always assume that $X$ is a real topological vector space and $Y$ is a topological vector space with an ordering cone $C$.

Now we consider the following vector optimisation problem:

$$
\text { (VOP) } \max \{f(x): x \in A\},
$$

where $A$ is a nonempty subset in $X$ and $f: A \rightarrow Y$ is a vector-valued function.

$E(f(A), C)$ denotes the set of all the efficient points of $f(A)$, and $E(A, f, C)$ denotes the set of all the efficient solutions of vector optimisation problem, that is,

$$
E(f(A), C)=\{y \in f(A):(y+C) \cap f(A)=y\}
$$


and

$$
E(A, f, C)=\{x \in A: f(x) \in E(f(A), C)\} .
$$

Let $F$ denote a set-valued map from $B \subset Y$ to $X$ with $F(y) \neq \emptyset$ for all $y \in B$.

We say that $F$ is lower semicontinuous at $y_{0} \in B$ if for any net $\left\{y_{\alpha}: \alpha \in I\right\}$ converging to $y_{0}$ and any $x_{0} \in F\left(y_{0}\right)$, there exists a net $\left\{x_{\alpha}: \alpha \in I\right\}$ such that $x_{\alpha} \in F\left(y_{\alpha}\right)$ and $\left\{x_{\alpha}: \alpha \in I\right\}$ converges to $x_{0}$. We say that $F$ is lower semicontinuous on $B \subset Y$ if $F$ is lower semicontinuous at every point $y \in B$.

Definition 2.1: Let $A \subset X$ be convex, and let $h$ be a real-valued function defined on $A$. Then $h$ is said to be

(a) quasiconcave on $A$ if $h\left(t x_{1}+(1-t) x_{2}\right) \geqslant \min \left\{h\left(x_{1}\right), h\left(x_{2}\right)\right\}$ for any $x_{1}, x_{2}$ $\in A, t \in(0,1)$;

(b) strictly quasiconcave on $A$ if $h\left(t x_{1}+(1-t) x_{2}\right)>\min \left\{h\left(x_{1}\right), h\left(x_{2}\right)\right\}$ for any $x_{1}, x_{2} \in A, h\left(x_{1}\right) \neq h\left(x_{2}\right), t \in(0,1)$.

Benson and Sun [2] introduced the following concepts:

DEFINITION 2.2: Let $f(x)=\left(f_{1}(x), f_{2}(x), \ldots, f_{p}(x)\right)^{T}$ be a $p$-dimensional continuous vector-valued function defined on the convex set $A \subset R^{n}$. Then $f$ is said to be

(a) quasiconcave on $A$ when the level set $M(y)=\{x \in A: f(x) \geqslant y\}$ is convex for any $y \in R^{p}$ satisfying $M(y) \neq \emptyset$;

(b) strictly quasiconcave on $A$ when $f$ is quasiconcave on $A$ and the set-valued mapping $M(y)=\{x \in A: f(x) \geqslant y\}$ is lower semicontinuous on $G$ $=\left\{y \in R^{p}: M(y) \neq \emptyset\right\}$.

REMARK 2.1. The concept of a strictly quasiconcave vector-valued function introduced by Benson and Sun is an important tool for studying the closedness of an efficient solution set. When $p=1$, the equivalence between Definition.2.2 (a) and Definition 2.1 (a) is well known. When $X=R^{n}$, Benson and Sun [2] pointed out if the real-valued function $f$ is continuous, then Definition 2.1 (b) is equivalent to Definition 2.2 (b). But for the general case, it is still an open question whether Definition 2.1 (b) is equivalent to Definition 2.2 (b).

Now we extend the above concepts to a topological vector space. In order to avoid any misunderstanding, we give the name of an $S$-strictly quasiconcave function.

Definition 2.3: Let $A$ be a nonempty convex subset of $X$. A vector-valued function $f: A \rightarrow Y$ is said to be

(a) quasiconcave on $A$ when the level set $M(y)=\{x \in A: f(x) \geqslant y\}$ is convex for any $y \in Y$ satisfying $M(y) \neq \emptyset$;

(b) $S$-strictly quasiconcave on $A$ when $f$ is quasiconcave on $A$ and the setvalued mapping $M(y)=\{x \in A: f(x) \geqslant y\}$ is lower semicontinuous on $G=\left\{y \in R^{p}: M(y) \neq \emptyset\right\}$. 
We also need the following concepts.

Definition 2.4: (See [6].) Let $Y$ be a topological vector lattice with the ordering cone $C$. A vector-valued function $f: A \subset X \rightarrow Y$ is said to be

(a) C-strictly quasiconcave when $f$ is quasiconcave and

$$
f\left(t x_{1}+(1-t) x_{2}\right) \in \inf \left\{f\left(x_{1}\right), f\left(x_{2}\right)\right\}+C \backslash\{0\}
$$

for any $x_{1}, x_{2} \in A, f\left(x_{1}\right) \neq f\left(x_{2}\right)$, and $t \in(0,1)$.

(b) $C$-strongly quasiconcave when $f$ is quasiconcave and

$$
f\left(t x_{1}+(1-t) x_{2}\right) \in \inf \left\{f\left(x_{1}\right), f\left(x_{2}\right)\right\}+\operatorname{int} C
$$

for any $x_{1}, x_{2} \in A, x_{1} \neq x_{2}$, and $t \in(0,1)$.

REMARK 2.2. It is easy to see that $f$ is quasiconcave if and only if

$$
f\left(t x_{1}+(1-t) x_{2}\right) \in \inf \left\{f\left(x_{1}\right), f\left(x_{2}\right)\right\}+C,
$$

for any $x_{1}, x_{2} \in A$, and $t \in(0,1)$.

Let $Y$ be a topological vector lattice with the ordering cone $C$. Let $e \in \operatorname{int} C$. Define a real-valued function from $Y$ to $R$ by

$$
g(y)=\sup \{t \in R: y \in t e+C\}, \quad y \in Y \text {. }
$$

This function is well defined and has the following properties.

LEMMA 2.1.

(i) $\min \left\{g\left(y_{1}\right), g\left(y_{2}\right)\right\} \leqslant g\left(\inf \left\{y_{1}, y_{2}\right\}\right)$;

(ii) $g$ is increasing, that is, if $y_{1} \leqslant y_{2}$, then $g\left(y_{1}\right) \leqslant g\left(y_{2}\right)$;

(iii) $g$ is strictly increasing, that is, if $y_{1}<y_{2}$, then $g\left(y_{1}\right)<g\left(y_{2}\right)$;

(iv) $g$ is continuous.

Proof: It is similar to the proof of [5, Lemma 1-4].

LEMMA 2.2 .

(i) $\inf \left\{y_{1}+u, y_{2}+u\right\}=u+\inf \left\{y_{1}, y_{2}\right\}$, for any $y_{1}, y_{2}, u \in Y$.

(ii) If $y_{1}, y_{2} \in \operatorname{int} C$, then $\inf \left\{y_{1}, y_{2}\right\} \in \operatorname{int} C$.

Proof: (i) is evident.

For (ii), let $y_{1}, y_{2} \in \operatorname{int} C$, then there exists a neighbourhood $U$ of 0 such that

$$
y_{i}+U \subset C, i=1,2
$$

For any $u \in U$, we have $y_{i}+u \in C, i=1,2$. Therefore,

$$
\inf \left\{y_{1}, y_{2}\right\}+u=\inf \left\{y_{1}+u, y_{2}+u\right\} \in C \text {. }
$$


It follows that

$$
\inf \left\{y_{1}, y_{2}\right\}+U \subset C .
$$

This means that $\inf \left\{y_{1}, y_{2}\right\} \in \operatorname{int} C$.

LEMma 2.3. If $f: A \subset X \rightarrow Y$ is an $S$-strictly quasiconcave vector-valued function, then $g \circ f$ is an $S$-strictly quasiconcave real-valued function, where $g$ is defined by (1).

Proof: First, we would like prove that $L(r)=\{x \in A: g \circ f(x) \geqslant r\}$ is convex for any scalar $r \in R$ satisfying $L(r) \neq \emptyset$. Let $x_{1}, x_{2} \in L(r)$, then

$$
g \circ f\left(x_{1}\right) \geqslant r, g \circ f\left(x_{2}\right) \geqslant r .
$$

Let $y=\inf \left\{f\left(x_{1}\right), f\left(x_{2}\right)\right\}$, then $f\left(x_{1}\right) \geqslant y, f\left(x_{2}\right) \geqslant y$, and $x_{1}, x_{2} \in\{x \in A: f(x) \geqslant y\}$. Since $f$ is quasiconcave, $\{x \in A: f(x) \geqslant y\}$ is convex. Therefore, we have

$$
t x_{1}+(1-t) x_{2} \in\{x \in A: f(x) \geqslant y\}, \quad \text { for all } t \in(0,1)
$$

that is,

$$
f\left(t x_{1}+(1-t) x_{2}\right) \geqslant y=\inf \left\{f\left(x_{1}\right), f\left(x_{2}\right)\right\} .
$$

It follows from (2), (3) and Lemma 2.1 that

$$
\begin{aligned}
r & \leqslant \min \left\{g \circ f\left(x_{1}\right), g \circ f\left(x_{2}\right)\right\} \leqslant g\left(\inf \left\{f\left(x_{1}\right), f\left(x_{2}\right)\right\}\right) \\
& \leqslant g \circ f\left(t x_{1}+(1-t) x_{2}\right), \quad \text { for all } t \in(0,1) .
\end{aligned}
$$

Hence, $t x_{1}+(1-t) x_{2} \in L(r)$, for all $t \in(0,1)$. This means that $L(r)$ is convex, and therefore, $g \circ f$ is quasiconcave.

Now we show that $L(r)$ is lower semicontinuous on $G^{\prime}=\{r \in R: L(r) \neq \emptyset\}$. Suppose that the net $\left\{r_{\alpha}: \alpha \in I\right\}$ converges to $r^{*}$ and $x^{*} \in L\left(r^{*}\right)$, then

$$
g \circ f\left(x^{*}\right) \geqslant r^{*}
$$

We define

$$
M(y)=\{x \in A: f(x) \geqslant y\}, \quad y \in Y
$$

and

$$
y_{\alpha}=f\left(x^{*}\right)+\left(r_{\alpha}-r^{*}\right) e, \text { for all } \alpha \in I .
$$

Thus, $y_{\alpha} \rightarrow f\left(x^{*}\right)$ and $x^{*} \in M\left(f\left(x^{*}\right)\right)$. Since $f$ is $S$-strictly quasiconcave, there exists a net $\left\{x_{\alpha}: \alpha \in I\right\}$ such that $x_{\alpha} \in M\left(y_{\alpha}\right)$ and $x_{\alpha} \rightarrow x^{*}$. We have $f\left(x_{\alpha}\right) \geqslant y_{\alpha}$. Since $g$ is increasing, we have

$$
g \circ f\left(x_{\alpha}\right) \geqslant g\left(y_{\alpha}\right)
$$


Noting that (4), (5) and

$$
\begin{aligned}
g\left(y_{\alpha}\right) & =g\left(f\left(x^{*}\right)+\left(r_{\alpha}-r^{*}\right) e\right) \\
& =\sup \left\{t \in R: f\left(x^{*}\right)+\left(r_{\alpha}-r^{*}\right) e \in t e+C\right\} \\
& =\sup \left\{t \in R: f\left(x^{*}\right) \in\left[t-\left(r_{\alpha}-r^{*}\right)\right] e+C\right\} \\
& =r_{\alpha}-r^{*}+g \circ f\left(x^{*}\right)
\end{aligned}
$$

we obtain

$$
g \circ f\left(x_{\alpha}\right) \geqslant g\left(y_{\alpha}\right)=r_{\alpha}-r^{*}+g \circ f\left(x^{*}\right) \geqslant r_{\alpha}-r^{*}+r^{*}=r_{\alpha}
$$

Hence, $x_{\alpha} \in L\left(r_{\alpha}\right), \alpha \in I$ and $x_{\alpha} \rightarrow x^{*}$. This implies $L(r)$ is lower semicontinuous on $G^{\prime}$. By Definition $2.3, g \circ f$ is $S$-strictly quasiconcave on $A$.

Benson and Sun [2] had the following result:

Let $h$ be a continuous real-valued function defined on the convex set $A \subset R^{n}$. If $h$ is quasiconcave on $A$ and $L(r)=\{x \in A: h(x) \geqslant r\}$ is a lower semicontinuous set-valued mapping on $G^{\prime}=\{r \in R: L(r) \neq \emptyset\}$, then $h$ is strictly quasiconcave on $A$.

In order to investigate the connectedness of an efficient solution set in a topological vector space, we need to extend the above result to a topological vector space.

LEMMA 2.4. Let $A$ be a convex subset of topological vector space $X$. If $h: A \rightarrow R$ is a continuous and $S$-strictly quasiconcave function, then $h$ is a strictly quasiconcave function.

Proof: Assume that $h$ is a continuous $S$-strictly quasiconcave real-valued function on $A$. Suppose to the contrary that $h$ is not strictly quasiconcave on $A$. Then there exist $x_{1}, x_{2} \in A$ with $h\left(x_{1}\right) \neq h\left(x_{2}\right)$ and $t_{0} \in(0,1)$ such that

$$
h\left(t_{0} x_{1}+\left(1-t_{0}\right) x_{2}\right) \leqslant \min \left\{h\left(x_{1}\right), h\left(x_{2}\right)\right\} .
$$

Let $x^{0}=t_{0} x_{1}+\left(1-t_{0}\right) x_{2}$ and $h\left(x_{1}\right)<h\left(x_{2}\right)$. We have

$$
h\left(x^{0}\right) \leqslant h\left(x_{1}\right)
$$

On the other hand, since $h$ is quasiconcave,

$$
h\left(x^{0}\right) \geqslant \min \left\{h\left(x_{1}\right), h\left(x_{2}\right)\right\} .
$$

We get

$$
h\left(x^{0}\right)=h\left(x_{1}\right)
$$

By the quasiconcavity of $h$, we have

$$
h\left(t x_{1}+(1-t) x^{0}\right) \geqslant \min \left\{h\left(x_{1}\right), h\left(x^{0}\right)\right\}=h\left(x^{0}\right), \quad \text { for all } \quad t \in(0,1) .
$$


First, we prove $h\left(t x_{1}+(1-t) x^{0}\right) \leqslant h\left(x^{0}\right)$ for all $t \in(0,1)$. Suppose to the contrary that there exists $t^{\prime} \in(0,1)$ such that

$$
h\left(t^{\prime} x_{1}+\left(1-t^{\prime}\right) x^{0}\right)>h\left(x^{0}\right) .
$$

Let $x_{0}=t^{\prime} x_{1}+\left(1-t^{\prime}\right) x^{0}$, then

$$
h\left(x_{0}\right)>h\left(x^{0}\right) .
$$

Obviously, there exists $t_{1} \in(0,1)$ such that

$$
x^{0}=t_{1} x_{0}+\left(1-t_{1}\right) x_{2} .
$$

By the quasiconcavity of $h$, we have

$$
h\left(x^{0}\right) \geqslant \min \left\{h\left(x_{0}\right), h\left(x_{2}\right)\right\} .
$$

If $h\left(x_{0}\right) \geqslant h\left(x_{2}\right)$, by (9), $h\left(x^{0}\right) \geqslant h\left(x_{2}\right)>h\left(x_{1}\right)$, which contradicts (6). If $h\left(x_{0}\right)$ $<h\left(x_{2}\right)$, by $(9)$, we have $h\left(x^{0}\right) \geqslant h\left(x_{0}\right)$, which contradicts (8). Hence,

$$
h\left(t x_{1}+(1-t) x^{0}\right) \leqslant h\left(x^{0}\right), \quad \text { for all } t \in(0,1) .
$$

By (7), (10) and (6), we have

$$
h\left(t x_{1}+(1-t) x^{0}\right)=h\left(x^{0}\right)=h\left(x_{1}\right), \quad \text { for all } t \in(0,1) .
$$

Next, we show that there exist $x^{*} \in\left(x_{1}, x^{0}\right)$ and a neighbourhood $U\left(x^{*}\right)$ of $x^{*}$ such that

$$
h(u) \leqslant h\left(x^{*}\right) \quad \text { for all } u \in U\left(x^{*}\right) .
$$

Since $h\left(x_{2}\right)>h\left(x_{1}\right),\left(1 / 2\left(h\left(x_{1}\right)+h\left(x_{2}\right)\right), h\left(x_{2}\right)+1\right)$ is an open neighbourhood of $h\left(x_{2}\right)$ and $h$ is continuous at $x_{2}$, there exists an open neighbourhood $U\left(x_{2}\right)$ of $x_{2}$ such that

$$
\frac{1}{2}\left(h\left(x_{1}\right)+h\left(x_{2}\right)\right)<h(x), \quad \text { for all } \quad x \in U\left(x_{2}\right) .
$$

Hence,

$$
h\left(x_{1}\right)<\frac{1}{2}\left(h\left(x_{1}\right)+h\left(x_{2}\right)\right)<h(x), \quad \text { for all } x \in U\left(\dot{x}_{2}\right) .
$$

Pick $x^{*} \in\left(x_{1}, x^{0}\right)=\left\{t x_{1}+(1-t) x^{0}: t \in(0,1)\right\}$ and $x^{* *} \in\left(x^{0}, x_{2}\right)=\left\{t x^{0}+(1-t) x_{2}\right.$ : $t \in(0,1)\}$ such that

$$
x^{0}=\frac{1}{2}\left(x^{*}+x^{* *}\right)
$$

By (11) and (12),

$$
h\left(x^{*}\right)<h(x), \quad \text { for all } x \in U\left(x_{2}\right)
$$


Let

$$
B=x^{0}+\cup\left\{\left\{t\left(x-x^{0}\right): x \in U\left(x_{2}\right)\right\}:-1 \leqslant t \leqslant 0\right\} .
$$

It is clear that $x^{0}+t\left(U\left(x_{2}\right)-x^{0}\right) \subset B$, for each $-1 \leqslant t \leqslant 0$.

To show $x^{*} \in \operatorname{int} B$. By $x^{0}=1 / 2\left(x^{*}+x^{* *}\right)$ and $x^{* *}=t x^{0}+(1-t) x_{2}$, where $0<t<1$, we have

$$
2 x^{0}-x^{*}=t x^{0}+(1-t) x_{2}
$$

Hence,

$$
x^{*}=-(1-t)\left(x_{2}-x^{0}\right)+x^{0} \in-(1-t)\left[U\left(x_{2}\right)-x^{0}\right]+x^{0} \subset B .
$$

This means that $x^{*} \in$ int $B$.

Since $h$ is continuous at $x^{*}$ and $\left(h\left(x^{*}\right)-1,1 / 2\left(h\left(x^{*}\right)+h\left(x_{2}\right)\right)\right)$ is an open neighbourhood of $h\left(x^{*}\right)$, there exists a neighbourhood $U\left(x^{*}\right)$ of $x^{*}$ such that $U\left(x^{*}\right) \subset B$ and

$$
h(u)<\frac{1}{2}\left(h\left(x^{*}\right)+h\left(x_{2}\right)\right), \quad \text { for all } u \in U\left(x^{*}\right) .
$$

This, together with $h\left(x^{*}\right)=h\left(x_{1}\right)$ and (12), yields that

$$
h(u)<h(x), \text { for all } u \in U\left(x^{*}\right) \text { and for all } x \in U\left(x_{2}\right) \text {. }
$$

Assume that there exists $u \in U\left(x^{*}\right)$ such that

$$
h(u)>h\left(x^{*}\right)
$$

It follows from $u \in U\left(x^{*}\right) \subset B$ that $u=t\left(x-x^{0}\right)+x^{0}$, where $-1 \leqslant t \leqslant 0$ and $x \in U\left(x_{2}\right)$. We have

$$
x^{0}=[1 /(1-t)] u+[-t /(1-t)] x
$$

Since $x^{*} \in\left(x_{1}, x^{0}\right)$ and by $(11), h\left(x^{*}\right)=h\left(x^{0}\right)$. By the quasiconcavity of $h,(13)$ and $x \in U\left(x_{2}\right)$

$$
h\left(x^{*}\right)=h\left(x^{0}\right) \geqslant \min \{h(u), h(x)\}=h(u),
$$

which contradicts (14). Hence, we have

$$
h(u) \leqslant h\left(x^{*}\right) \quad \text { for all } \quad u \in U\left(x^{*}\right) .
$$

Finally, we show that $h$ is not $S$-strictly quasiconcave on $A$. Let $r^{*}=h\left(x^{*}\right)$, then

$$
x^{*} \in L\left(r^{*}\right)=\left\{x \in A: h(x) \geqslant r^{*}\right\}
$$

Let $r_{n}=1 / n\left[h\left(x_{2}\right)\right]+(1-1 / n) h\left(x^{*}\right)$. Thus, $r_{n} \rightarrow r^{*}$.

It is clear that $\left\{r_{n}\right\}$ is a net and $h\left(x_{2}\right) \geqslant r_{n}$. We know that $\left\{r_{n}\right\} \subset G^{\prime}=\{r \in R$ : $L(r) \neq \emptyset\}$ 
For any sequence $\left\{x_{n}\right\}$ satisfying $x_{n} \in L\left(r_{n}\right)$, we have

$$
h\left(x_{n}\right) \geqslant r_{n}>r^{*}=h\left(x^{*}\right) .
$$

By (15) and (16), $\left\{x_{n}\right\}$ can not converge to $x^{*}$. Hence, $L(r)$ is not lower semicontinuous at $r^{*} \in G^{\prime}$. This means that the real-valued function $h$ is not $S$-strictly quasiconcave on $A$.

This contradicts the assumption that $h$ is an $S$-strictly quasiconcave function on $A$. Therefore, $h$ is strictly quasiconcave.

\section{Relation among Various Quasiconcavities}

The concept of an $S$-strictly quasiconcave vector-valued function is a key tool for us to study the closedness and the connectedness of an efficient solution set. First we discuss relation among $S$-strictly quasiconcave, $C$-strongly quasiconcave, and $C$-strictly quasi-concave vector-valued functions.

THEOREM 3.1. Let $Y$ be a topological vector lattice with the ordering cone $C$. If $f: A \subset X \rightarrow Y$ is a continuous and $C$-strongly quasiconcave function and $A$ is a compact convex set, then $f$ is $S$-strictly quasiconcave on $A$.

Proof: Suppose that $f$ is not an $S$-strictly quasiconcave function on $A$, then $M(y)=\{x \in A: f(x) \geqslant y\}$ is not lower semicontinuous on $G=\{y: M(y) \neq \emptyset\}$. Hence, there exists $y^{*} \in G$ such that $M(y)$ is not lower semicontinuous at $y^{*}$. By the definition, there exist $x^{*} \in M\left(y^{*}\right)$, an open neighbourhood $U\left(x^{*}\right)$ of $x^{*}$ and a net $\left\{y_{\alpha}: \alpha \in I\right\} \subset G$ such that $y_{\alpha} \rightarrow y^{*}$ and

$$
M\left(y_{\alpha}\right) \cap U\left(x^{*}\right)=\emptyset, \quad \text { for all } \quad \alpha \in I .
$$

Pick $x_{\alpha} \in M\left(y_{\alpha}\right)$, for each $\alpha \in I$. We have

$$
f\left(x_{\alpha}\right) \geqslant y_{\alpha}, \quad \text { for all } \alpha \in I .
$$

Since $\left\{x_{\alpha}\right\} \subset A$ and $A$ is compact, we can assume that $x_{\alpha} \rightarrow x_{0} \in A$. Taking the limit on both sides of $(18)$, we get

$$
f\left(x_{0}\right) \geqslant y^{*}
$$

since $f$ is continuous and $y_{\alpha} \rightarrow y^{*}$.

If $x_{0}=x^{*}$, then $x_{\alpha} \rightarrow x^{*}$. Since $U\left(x^{*}\right)$ is a neighbourhood of $x^{*}$, there exists $\alpha_{0} \in I$ such that $x_{\alpha} \in U\left(x^{*}\right)$, for all $\alpha \geqslant \alpha_{0}$. Hence,

$$
x_{\alpha} \in M\left(y_{\alpha}\right) \cap U\left(x^{*}\right),
$$

which contradicts (17). 
If $x_{0} \neq x^{*}$, since $f$ is $C$-strongly quasiconcave and $f\left(x_{0}\right) \geqslant y^{*}$ and $f\left(x^{*}\right) \geqslant y^{*}$, we have

$$
f\left[(1 / k) x_{0}+(1-1 / k) x^{*}\right] \in \inf \left\{f\left(x_{0}\right), f\left(x^{*}\right)\right\}+\operatorname{int} C \in y^{*}+\operatorname{int} C .
$$

Let $u_{k}=(1 / k) x_{0}+(1-1 / k) x^{*}$, then $u_{k} \rightarrow x^{*}$ as $k \rightarrow \infty$, and

$$
f\left(u_{k}\right)-y^{*} \in \operatorname{int} C, \text { for all } k=1,2, \ldots
$$

Pick $k$ such that $u_{k} \in U\left(x^{*}\right)$. For this $k$, it follows from (19) that there exists a symmetric neighbourhood $U(0)$ of 0 such that

$$
f\left(u_{k}\right)-y^{*}+U(0) \subset C .
$$

Since $y_{\alpha} \rightarrow y^{*}$, there exists an $\alpha$ such that

$$
y_{\alpha}-y^{*} \in U(0)
$$

Therefore,

$$
f\left(u_{k}\right)-y_{\alpha}=f\left(u_{k}\right)-y^{*}-\left(y_{\alpha}-y^{*}\right) \in f\left(u_{k}\right)-y^{*}+U(0) \subset C .
$$

We obtain that $f\left(u_{k}\right) \geqslant y_{\alpha}$, and hence $u_{k} \in M\left(y_{\alpha}\right) \cap U\left(x^{*}\right)$, which contradicts (17). Therefore, $f$ is $S$-strictly quasiconcave.

REMARK 3.1. It is clear that an $S$-strictly quasiconcave function is not necessarily $C$ strongly quasiconcave.

In order to prove that an $S$-strictly quasiconcave function is a $C$-strictly quasiconcave function when the ordering cone $C$ has a nonempty interior, we need the following lemma.

LEMMA 3.1. Let $Y$ be a topological vector lattice with the ordering cone $C$. If $y_{1}, y_{2} \in \operatorname{int} C, y_{1} \neq y_{2}$, then there exists a function

$$
g(y)=\sup \{t \in R: y \in t e+C\}, \quad y \in Y,
$$

such that $g\left(y_{1}\right) \neq g\left(y_{2}\right)$, where $e \in \operatorname{int} C$.

Proof: By $y_{1}, y_{2} \in \operatorname{int} C$, and $y_{1} \neq y_{2}$, we have either $y_{1} \leqslant y_{2}$, or $y_{1} \notin y_{2}+C$ and $y_{2} \notin y_{1}+C$. We can pick $y^{\prime} \in y_{1}+\operatorname{int} C$ and $y^{\prime \prime} \in y_{2}-\operatorname{int} C$, such that $y^{\prime}, y^{\prime \prime} \in \operatorname{int} C$ and $y_{1} \notin y^{\prime \prime}+C$.

Let $e=\inf \left\{y^{\prime}, y^{\prime \prime}\right\}$. By Lemma 2.2,e $\in$ int $C$. Denote

$$
g(y)=\sup \{t \in R: y \in t e+C\}, \quad y \in Y .
$$

It is clear that $g$ satisfies the properties stated in Lemma 2.1. Since $e=\inf \left\{y^{\prime}, y^{\prime \prime}\right\}$ $\leqslant y^{\prime \prime}<y_{2}$ and $g$ is strictly increasing, $1=g(e)<g\left(y_{2}\right)$. 
If $g\left(y_{1}\right)<1$, then $g\left(y_{1}\right) \neq g\left(y_{2}\right)$. Suppose that $g\left(y_{1}\right) \geqslant 1$, then $y_{1} \in g\left(y_{1}\right) e+C$, therefore, $y_{1} \in e+C$. We obtain

$$
e \leqslant \inf \left\{y_{1}, y^{\prime \prime}\right\} \leqslant \inf \left\{y^{\prime}, y^{\prime \prime}\right\}=e,
$$

Hence,

$$
e=\inf \left\{y_{1}, y^{\prime \prime}\right\}=\inf \left\{y^{\prime}, y^{\prime \prime}\right\} .
$$

Since $y_{1}<y^{\prime}$ and $y_{1} \notin y^{\prime \prime}+C$, we have

$$
e=\inf \left\{y_{1}, y^{\prime \prime}\right\}<y^{\prime}, e \leqslant y^{\prime \prime} \text { and } e \neq y^{\prime \prime} .
$$

Therefore, $y^{\prime}=e+c_{0}$ and $y^{\prime \prime}=e+c$, where $c_{0} \in \operatorname{int} C, c \in C \backslash\{0\}$.

By Lemma 2.2, we have

$$
\inf \left\{y^{\prime}, y^{\prime \prime}\right\}=\inf \left\{e+c_{0}, e+c\right\}=\inf \left\{c_{0}, c\right\}+e .
$$

By $c_{0} \in \operatorname{int} C$, there exists a symmetric neighbourhood $U(0)$ of 0 such that $c_{0}+U(0) \subset C$. Since there exists $0<t<1$ such that $-t c \in U(0)$, we have

$$
\inf \left\{c_{0}, c\right\} \geqslant \inf \left\{c_{0}-t c+t c, t c\right\} .
$$

Since $c_{0}-t c \in c_{0}+U(0) \subset C$,

$$
c_{0}-t c+t c \geqslant t c
$$

and

$$
\inf \left\{c_{0}, c\right\} \geqslant t c \neq 0
$$

Therefore,

$$
\inf \left\{y^{\prime}, y^{\prime \prime}\right\}-e=\inf \left\{c_{0}, c\right\} \in C \backslash\{0\} .
$$

This contradicts that $e=\inf \left\{y^{\prime}, y^{\prime \prime}\right\}$. Hence, we have $g\left(y_{1}\right)<1<g\left(y_{2}\right)$. The proof is completed.

REMARK 3.2. It is easy to see that for any fixed $b \in Y$, the function $h(\cdot)=f(\cdot)$ $+b$ is $S$-strictly ( $C$-strictly) quasiconcave if and only if $f(\cdot)$ is $S$-strictly ( $C$-strictly) quasiconcave. If int $C \neq \emptyset, A$ is a compact convex subset of $X$ and $f: A \rightarrow Y$ is continuous, then there exist $c \in \operatorname{int} C$, a neighbourhood $U(0)$ of 0 and $t>0$ such that

$$
c+U(0) \subset \operatorname{int} C
$$

and

$$
f(A) \subset t U(0)
$$

Therefore,

$$
t c+f(A) \subset t c+t U(0)=t(c+U(0)) \subset t \operatorname{int} C \subset \operatorname{int} C .
$$

Let $h(x)=t c+f(x), x \in A$. 
Then, $h(A) \subset \operatorname{int} C$. As mentioned above, $h(x)$ and $f(x)$ have the same $S$-strictly ( $C$-strictly) quasiconcavity on $A$.

THEOREM 3.2. Let $Y$ be a topological vector lattice with the ordering cone $C$. If int $C \neq \emptyset, A$ is a compact convex subset of $X$, and if $f: A \subset X \rightarrow Y$ is continuous and $S$-strictly quasiconcave on $A$, then $f$ is $C$-strictly quasiconcave on $A$.

Proof: By Remark 3.2, we can assume that $f(A) \subset$ int $C$. If $f$ is not $C$-strictly quasi-concave, then there exist $x_{1}, x_{2} \in A$ with $f\left(x_{1}\right) \neq f\left(x_{2}\right)$ and $t_{0} \in(0,1)$ such that

$$
f\left(t_{0} x_{1}+\left(1-t_{0}\right) x_{2}\right) \notin \inf \left\{f\left(x_{1}\right), f\left(x_{2}\right)\right\}+C \backslash\{0\}
$$

Since $f$ is quasiconcave and by Remark 2.2,

$$
f\left(t_{0} x_{1}+\left(1-t_{0}\right) x_{2}\right) \in \inf \left\{f\left(x_{1}\right), f\left(x_{2}\right)\right\}+C .
$$

It follows that

$$
f\left(t_{0} x_{1}+\left(1-t_{0}\right) x_{2}\right)=\inf \left\{f\left(x_{1}\right), f\left(x_{2}\right)\right\} .
$$

Let $x^{0}=t_{0} x_{1}+\left(1-t_{0}\right) x_{2}$, then

$$
f\left(x^{0}\right)=\inf \left\{f\left(x_{1}\right), f\left(x_{2}\right)\right\}
$$

Since $f\left(x_{1}\right) \neq f\left(x_{2}\right)$ and $f\left(x_{1}\right), f\left(x_{2}\right) \in \operatorname{int} C$, by Lemma 3.1, there exists

$$
g(y)=\sup \{t \in R: y \in t e+C\}, \quad y \in Y,
$$

where $e \in \operatorname{int} C$ and $g \circ f\left(x_{1}\right) \neq g \circ f\left(x_{2}\right)$. By Lemma 2.1, we have

$$
g\left(\inf \left\{f\left(x_{1}\right), f\left(x_{2}\right)\right\}\right)=g \circ f\left(x^{0}\right) \geqslant \min \left\{g \circ f\left(x_{1}\right), g \circ f\left(x_{2}\right)\right\} .
$$

On the other hand, we have $f\left(x^{0}\right) \leqslant f\left(x_{1}\right)$ and $f\left(x^{0}\right) \leqslant f\left(x_{2}\right)$. Since $g$ is increasing, we have $g \circ f\left(x^{0}\right) \leqslant g \circ f\left(x_{1}\right)$ and $g \circ f\left(x^{0}\right) \leqslant g \circ f\left(x_{2}\right)$. Hence,

$$
g \circ f\left(x^{0}\right) \leqslant \min \left\{g \circ f\left(x_{1}\right), g \circ f\left(x_{2}\right)\right\} .
$$

From (20) and (21), we obtain

$$
g \circ f\left(x^{0}\right)=g \circ f\left(t_{0} x_{1}+\left(1-t_{0}\right) x_{2}\right)=\min \left\{g \circ f\left(x_{1}\right), g \circ f\left(x_{2}\right)\right\} .
$$

By Lemma 2.3 and Lemma 2.4, $g \circ f$ is a strictly quasiconcave real-valued function. Noticing that $g \circ f\left(x_{1}\right) \neq g \circ f\left(x_{2}\right)$, we have

$$
g \circ f\left(t_{0} x_{1}+\left(1-t_{0}\right) x_{2}\right)>\min \left\{g \circ f\left(x_{1}\right), g \circ f\left(x_{2}\right)\right\}
$$

which contradicts (22). Hence, $f$ is $C$-strictly quasiconcave on $A$. 
The following example shows that a $C$-strictly quasiconcave vector-valued function is not necessarily strictly quasiconcave.

EXAMPLE 3.1. Let $C=R_{+}^{2} \subset R^{2}$ and $f=\left(f_{1}, f_{2}\right):[-1,1] \rightarrow R^{2}$, where

$$
f_{1}(x)=\left\{\begin{aligned}
-x, & x \in[-1,0], \quad f_{2}(x)=-x . \\
0, & x \in(0,1],
\end{aligned}\right.
$$

It is clear that $f$ is $C$-strictly quasiconcave. If $f$ is $S$-strictly quasiconcave, by [2, Theorem 2.4.], $f_{1}$ must be a strictly quasiconcave real-valued function. But, $f_{1}$ is not a strictly quasiconcave function, which is a contradiction. Therefore, $f$ is not $S$-strictly quasiconcave.

\section{Closedness and CONNECTEDness}

THEOREM 4.1. Let $A$ be a compact convex subset of topological vector space $X$ and let $Y$ be a topological vector lattice with the ordering cone $C$. Assume that $f: A \rightarrow Y$ is a continuous $S$-strictly quasiconcave function, then $E(f(A), C)$ and $E(A, f, C)$ are closed.

Proof: Let a net $\left\{y_{\alpha}: \alpha \in I\right\} \subset E(f(A), C)$ and $y_{\alpha} \rightarrow y_{0}$. Since $f$ is continuous and $A$ is a compact set, $f(A)$ is compact. Since $C$ is a closed convex pointed cone, the topology of $Y$ is Hausdorff (see [9]), so $f(A)$ is closed. Thus, there exists $x_{0} \in A$ such that $y_{0}=f\left(x_{0}\right) \in f(A)$. If $y_{0} \notin E(f(A), C)$, then there exists $x^{0} \in A$ such that

$$
f\left(x^{0}\right) \geqslant f\left(x_{0}\right), f\left(x^{0}\right) \neq f\left(x_{0}\right) .
$$

Let

$$
M(y)=\{x \in A: f(x) \geqslant y\}, \quad y \in Y .
$$

We have $M\left(f\left(x_{0}\right)\right) \neq \emptyset$. Since $f$ is $S$-strictly quasiconcave, by Definition $2.3, M(y)$ is lower semicontinuous at $f\left(x_{0}\right)$. Noticing that $y_{\alpha} \rightarrow y_{0}=f\left(x_{0}\right)$ and $x^{0} \in M\left(f\left(x_{0}\right)\right)$, by the lower semicontinuity of $M$, there exists a net $\left\{x_{\alpha}: \alpha \in I\right\}$ such that $x_{\alpha} \in M\left(y_{\alpha}\right)$ and $x_{\alpha} \rightarrow x^{0}$. We have

$$
f\left(\left(x_{\alpha}\right)\right) \geqslant y_{\alpha}, \quad \text { for all } \quad \alpha \in I .
$$

Since $f\left(x^{0}\right) \neq f\left(x_{0}\right)$ and $Y$ is Hausdorff, there exist a neighbourhood $U\left(f\left(x_{0}\right)\right)$ of $f\left(x_{0}\right)$ and a neighbourhood $U\left(f\left(x^{0}\right)\right)$ of $f\left(x^{0}\right)$ such that

$$
U\left(f\left(x^{0}\right)\right) \cap U\left(f\left(x_{0}\right)\right)=\emptyset .
$$

Since $y_{\alpha} \rightarrow y_{0}=f\left(x_{0}\right)$ and $f\left(x_{\alpha}\right) \rightarrow f\left(x^{0}\right)$, there exists $\beta \in I$ such that

$$
f\left(x_{\alpha}\right) \in U\left(f\left(x^{0}\right)\right) \text { and } y_{\alpha} \in U\left(f\left(x_{0}\right)\right) .
$$


By (24) and (23),

$$
f\left(x_{\alpha}\right) \neq y_{\alpha} \quad \text { and } \quad f\left(x_{\alpha}\right) \geqslant y_{\alpha} .
$$

This follows that $y_{\alpha} \notin E(f(A), C)$, which contradicts that $y_{\alpha} \in E(f(A), C)$. Hence, $E(f(A), C)$ is closed. It is easy to see that $E(A, f, C)$ is also closed.

Theorem 4.1 is a generalisation of [2, Theorem 3.3$]$ which is one of the main result in [2]. The conditions of Theorem 4.1 are general and our proof is direct.

LEMMA 4.1. ([6]) Let $A$ be a compact convex subset of topological vector space $X$. Let $Y$ be a topological vector lattice with ordering cone $C$. Assume that $f: A \rightarrow Y$ is a continuous, $C$-strictly quasiconcave function and $E(f, A, C)$ is closed. Then $E(f, A, C)$ is closed and connected.

Combining Theorem 4.1, Lemma 4.1 and Theorem 3.2, we can immediately get the following theorem.

THEOREM 4.2. Let $A$ be a compact convex subset of topological vector space $X$ and $Y$ be a topological vector lattice with the ordering cone $C$. Assume that $f: A \rightarrow Y$ is a continuous $S$-strictly quasiconcave function and int $C \neq \emptyset$, then $E(f, A, C)$ is closed and connected.

\section{REFERENCES}

[1] J. Benoist, 'Connectedness of the efficient set for strictly quasiconcave sets', J. Optim. Theory Appl. 96 (1998), 627-654.

[2] H.P. Benson and E.J. Sun, 'New closedness results for efficient sets in multiple objective mathematical programming', J. Math. Anal. Appl. 238 (1999), 277-296.

[3] E.U. Choo, S. Schaible and K.P. Chew, 'Connectedness of the efficient set in three-criteria quasiconcave programming', Cahiers Centre Études Rech. Opér. 27 (1985), 213-220.

[4] A. Daniilidis, N. Hadjisavvas and S. Schaible, 'Connectedness of the efficient set for three objective quasiconcave maximization problems', J. Optim. Theory Appl. 93 (1997), 517-524.

[5] W.T. Fu, 'On the closedness and connectedness of the set of efficient points', (in Chinese), J. Math. Res. Exposition 14 (1994), 89-96.

[6] W.T. Fu and K.P. Zhou, 'Connectedness of the efficient solution sets for a strictly path quasiconvex programming problem', Nonlinear Anal. 21 (1993), 903-910.

[7] W.T. Fu and K.P. Zhou, 'The connectedness of the efficient solution sets for a strongly quasiconvex multiobjective optimization in infinite dimensional spaces', (in Chinese), Acta Math. Appl. Sinica 18 (1995), 140-146.

[8] Y.D. Hu and E.J. Sun, 'Connectedness of the efficient set in strictly quasiconcave vector maximization', J. Optim. Theory Appl. 78 (1993), 613-622.

[9] G. Jameson, Ordered linear spaces, Lecture Notes in Mathematics 141 (Springer-Verlag, Berlin, 1970).

[10] H.H. Schaefer, Topological vector spaces (Springer-Verlag, New York, 1970). 
[11] S. Schaible, 'Bicriteria quasiconcave programs', Cahiers Centre Études Rech. Opér. 25 (1983), 93-101.

[12] E.J. Sun, 'On the connectedness of the efficient set for strictly quasiconvex vector minimization problems', J. Optim. Theory Appl. 89 (1996), 475-481.

[13] P.L. Yu and M. Zeleny, 'The set of all nondominated solutions in linear cases and a multicriteria simplex method', J. Math. Anal. Appl. 49 (1975), 430-468.

Institute of Systems Science

Academy of Mathematics and Systems Sciences

Chinese Academy of Sciences

Beijing 100080

China

e-mail: donghb@amss.ac.cn

Institute of Systems Science

Academy of Mathematics and Systems Sciences

Chinese Academy of Sciences

Beijing 100080

China

e-mail: sywang@iss02.iss.ac.cn

and

School of Business Administration

Hunan University

Changsha

Hunan 410082

China

e-mail: sywang@hnu.edu.cn
Department of Mathematics

Nanchang University

Nanchang 330047

China

e-mail: ncxhgong@263.ne

Department of Statistics and Operations Research Universidade de Santiago 15782 Santiago de Compostela

Spain

e-mail: coladas@usc.es 